\title{
Strong and weak interactions in Ghahramany's integrated nuclear binding energy formula
}

Seshavatharam UVS ( $\nabla$ seshavatharam.uvs@gmail.com )

I-SERVE

Lakshminarayana S

Andhra University

\section{Research Article}

Keywords: Strong interaction, Electroweak interaction, Ghahramany's integrated nuclear binding energy formula

Posted Date: August 30th, 2021

DOl: https://doi.org/10.21203/rs.3.rs-855034/v1

License: (1) This work is licensed under a Creative Commons Attribution 4.0 International License. Read Full License 


\title{
STRONG AND WEAK INTERACTIONS IN GHAHRAMANY'S INTEGRATED NUCLEAR BINDING ENERGY FORMULA
}

\author{
U.V.S. Seshavatharam ${ }^{1}$ and S. Lakshminarayana ${ }^{2}$ \\ ${ }^{1}$ Honorary faculty, I-SERVE, Survey no-42, Hitech city, Hyderabad-84, Telangana, INDIA \\ ${ }^{2}$ Dept. of Nuclear Physics, Andhra University, Visakhapatnam-03,AP, INDIA \\ Emails: seshavatharam.uvs@gmail.com (and) 1nsrirama@gmail.com \\ Orcid numbers : 0000-0002-1695-6037 (and) 0000-0002-8923-772X
}

\begin{abstract}
By modifying Ghahramany's integrated nuclear binding energy formula with strong and weak interactions, it is possible to approximate the nuclear binding energy of isotopes with one unique energy coefficient and four terms. Considering even-odd corrections, shell corrections and other microscopic corrections, it seems possible to improve the accuracy with a clear physical basis. Based on our recent work and the proposed formula, we are very confident to say that, electroweak interaction plays a vital role in fixing the nuclear binding energy.
\end{abstract}

Keywords: Strong interaction; Electroweak interaction; Ghahramany's integrated nuclear binding energy formula;

\section{Introduction}

Semi empirical mass formulae (SEMF) are having a crucial role in understanding nuclear structure [1-4] and constitute a minimum of 5 terms with five different energy coefficients. Three major disadvantages of SEMF are: 1) Five energy coefficients are having arbitrary origin. 2) Role of strong and weak interactions is found to be missing. 3) Energy coefficients are having no particular relation with quarks and their structure. In this context, Ghahramany and team members [5-9] developed a very simple nuclear binding energy formula based on quark-gluon plasma. For increasing its effectiveness towards isotopes, in this paper, we try to include strong and weak interaction features [10,11] in Ghahramany's integrated nuclear binding energy formula (GINBEF).

\section{Ghahramany's Integrated nuclear model}

According to Ghahramany and team members, up and down quarks play a crucial role in understanding nuclear binding energy. Their unified views are:

1) The nuclear binding energy is of the order of $1 \%$ of the energy of the total rest mass of the constituent nucleons.

2) The binding energy depends upon the volume of the quark-gluon soup within the nuclei and is proportional to $3 \mathrm{~A}$ where $\mathrm{A}$ is the mass number.

3) Due to the asymmetric distribution of up and down quarks and also the existence of the Coulomb force between them, binding energy depends upon terms such as $\frac{N^{2}-Z^{2}}{Z}$. 
44 For $A \geq 5$, different binding energy formulae proposed by Ghahramany and team members are as

45

46

47

48

49

51 follows. In terms of up and down quarks,

$$
\begin{aligned}
& \left.B E \cong\left\{3 A-\frac{\left(N^{2}-Z^{2}\right)+\delta(N-Z)}{3 Z}-9\right\}\left(\frac{330 \mathrm{MeV}}{100}\right)\right\} \\
& \text { where, }\left\{\begin{array}{l}
\delta(N-Z)=0 \text { for } Z \neq N \\
\delta(N-Z)=1 \text { for } Z=N
\end{array}\right.
\end{aligned}
$$

With reference to nucleon rest energy,

$$
\begin{gathered}
\left.B E \cong\left\{A-\frac{\left(N^{2}-Z^{2}\right)+\delta(N-Z)}{3 Z}-3\right\}\left(\frac{m_{n} c^{2}}{90 \text { to } 100}\right) \mathrm{MeV}\right\} \\
\text { where, } m_{n} c^{2}=\text { Rest energy of nucleon } \\
\qquad\left\{\begin{array}{l}
\delta(N-Z)=0 \text { for } Z \neq N \\
\delta(N-Z)=1 \text { for } Z=N
\end{array}\right.
\end{gathered}
$$

More advanced formula is,

$$
\left.B E \cong\left\{A-\frac{\left(N^{2}-Z^{2}\right)+\delta(N-Z)}{3(Z-k)}-3\right\}\left(\frac{A^{n+s} m_{n} c^{2}}{126(Z-k)}\right) \mathrm{MeV}\right)
$$

where, $m_{n} c^{2}=$ Rest energy of nucleon

$$
\begin{aligned}
& n \cong 0.87 \text { to } 0.88, k \cong\left\{\begin{array}{l}
2 \text { for } Z \leq 118 \\
0 \text { for } Z>118
\end{array}\right. \\
& s \cong\left\{\begin{array}{l}
0.0003 \text { for even }(N, Z) \\
-0.0003 \text { for odd }(N, Z)
\end{array}\right.
\end{aligned}
$$

\section{Understanding the role of electroweak interaction in nuclear binding energy scheme}

With reference to strong and weak interactions in our recently published papers, we have developed a simple formula for estimating nuclear binding energy with one unique coefficient of magnitude $10.1 \mathrm{MeV}$. In this paper, we made an attempt to include strong and weak interactions in GINBEF. It may be noted that, single variable binding energy constant and 3 simple terms play an interesting role in GINBEF. By considering strong and weak interactions, we have improved the workability of GINBEF applicable for isotopes and it needs further study with respect to $Z \approx N$.

By considering electroweak interaction, in nuclear binding energy scheme, we noticed that,

1) Unique binding energy coefficient is interconnected with basic nuclear potential, reciprocal of the strong coupling constant [12] and up and down quark rest masses [12]. (See section 9).

2) All the nucleons are not participating in nuclear binding energy. 
3) Electroweak interaction plays a vital role in deciding the number of non-participating nucleons in the form of $[1+0.0019 A \sqrt{N Z}]$.

4) Electroweak interaction decides the stable mass numbers of $Z$ in the form of $A_{s} \cong 2 Z+0.0016(2 Z)^{2} \cong 2 Z+0.0064 Z^{2}$.

5) With reference to pions and electro weak bosons, number 0.0016 can be expressed with a relation of the form, $\left(\frac{\sqrt{\left(m_{\pi} c^{2}\right)^{0}\left(m_{\pi} c^{2}\right)^{ \pm}}}{\sqrt{\left(m_{z} c^{2}\right)^{0}\left(m_{w} c^{2}\right)^{ \pm}}}\right) \cong\left(\frac{\sqrt{134.98 \times 139.57} \mathrm{MeV}}{\sqrt{80379.0 \times 91187.6} \mathrm{MeV}}\right) \cong 0.0016032$.

6) Stable mass number of $Z$ plays an interesting role in reducing the binding energy of isotopes in the form of $\frac{\left(A_{s}-A\right)^{2}}{A_{s}}$.

7) Binding energy decreases with increasing surface area and increasing radius with an effective term of the form $\left(A^{2 / 3} / A^{1 / 3}\right) \cong A^{1 / 3}$.

Binding energy can be approximated with a relation of the form,

$$
B E \cong\left\{A-[1+0.0019 A \sqrt{N Z}]-A^{1 / 3}-\frac{\left(A_{s}-A\right)^{2}}{A_{s}}\right\}(10.1 \mathrm{MeV})
$$

By replacing the number 0.0019 with 0.0016 , binding energy can be approximated with a relation of the form,

$$
B E \cong\left\{A-A^{1 / 3}-\frac{\left(A_{s}-A\right)^{2}}{A_{s}}\right\}(10.1 \mathrm{MeV})-[1+0.0016 A \sqrt{N Z}](11.9 \mathrm{MeV})
$$

\section{On the role of electroweak interaction in Integrated nuclear binding energy formula}

Based on the above relations, we make an attempt to include strong and weak interactions in GINBEF. For $Z \geq 3$, it can be expressed in the following way.

$$
\begin{aligned}
B E \cong & \left\{A-\left[0.00173 \sqrt{Z N} \sqrt{A A_{s}}\right]-\left[\left(0.118 \frac{\left(N^{2}-Z^{2}\right)}{\sqrt{A A_{s}}}\right)+\left(\frac{\left(A-A_{s}\right)^{2}}{\sqrt{A A_{s}}}\right)\right]-\left[\sqrt{\frac{A}{Z}}+\frac{\sqrt{A}}{2}\right]\right) 10.3 \mathrm{MeV} \\
& \text { where } A_{s} \cong 2 Z+0.0064 Z^{2}
\end{aligned}
$$

With reference to various semi empirical mass formulae and with trial \& error we have developed this relation. It is having many advantages compared to Ghahramany's integrated nuclear model. In this relation,

1) Second term can be considered as a consequence of electroweak interaction.

2) Third term can be considered as a consequence of Coulombic and Asymmetry.

3) Fourth term can be considered as a correction factor associated with neutron-proton ratio.

As a whole, accuracy depends on two numbers 0.00173 and 0.118 , one energy coefficient of 10.3 
107

$\mathrm{MeV}$ and selection of stable mass number of $\mathrm{Z}$. With a computer program and fitting the estimated data with actual binding energies, things can be improved. By considering even-odd corrections, shell corrections and other microscopic corrections, relation (6) can be reviewed and refined with a clear physical background. See Table 1 for the binding energy of isotopes of $Z=50$. To compare the data prepared with relations (4), (5) and (6), we have taken the following three semi empirical mass formulae as references.

$$
B E_{\text {Refl }} \cong\left\{\begin{array}{c}
{[(A \times 15.36)]-\left[\left(A^{2 / 3} \times 16.32\right)\right]-\left[\left(\frac{Z^{2}}{A^{1 / 3}}\right) 0.6929\right]} \\
-\left[\frac{((A / 2)-Z)^{2}}{A} \times 90.46\right] \pm\left(\frac{11.32}{\sqrt{A}}\right)
\end{array}\right\} \mathrm{MeV}
$$

$$
\text { Ref. } \rightarrow \text { https://fdocuments.in/document/nuclear-and-particle-physics.html }
$$

$$
B E_{\mathrm{Ref} 2} \cong\left(a_{v} * A\right)-\left(a_{s} * A^{2 / 3}\right)-\left(a_{c} * \frac{Z *(Z-1)}{A^{1 / 3}}\right)-\left(a_{a} * \frac{(A-2 Z)^{2}}{A}\right) \pm\left(\frac{a_{p}}{\sqrt{A}}\right)
$$

$$
\text { where }\left\{\begin{array}{l}
a_{v} \cong 15.8 \mathrm{MeV} ; a_{s} \cong 18.3 \mathrm{MeV} ; a_{c} \cong 0.714 \mathrm{MeV} ; \\
a_{a} \cong 23.2 \mathrm{MeV} ; a_{p} \cong 12.0 \mathrm{MeV} ;
\end{array}\right.
$$

Ref. $\rightarrow$ https://en.wikipedia.org/wiki/Semi-empirical_mass_formula

\begin{tabular}{|c|c|c|c|c|c|c|c|c|c|}
\hline $\mathrm{Z}=50$ & $\mathrm{~N}$ & $\mathrm{~A}$ & $\begin{array}{c}\text { Estimated } \\
\text { stable } \\
\text { mass } \\
\text { number }\end{array}$ & $\begin{array}{c}\text { Ref_1.BE } \\
(\mathrm{MeV}) \\
\text { Relation-7 }\end{array}$ & $\begin{array}{c}\text { Ref_2.BE } \\
(\mathrm{MeV}) \\
\text { Relation-8 }\end{array}$ & $\begin{array}{c}\text { Ref3.BE } \\
(\mathrm{MeV}) \\
\text { Relation-9 }\end{array}$ & $\begin{array}{c}\text { Est_1 BE } \\
(\mathrm{MeV}) \\
\text { Relation-4 }\end{array}$ & $\begin{array}{c}\text { Est_2 BE } \\
(\mathrm{MeV}) \\
\text { Relation-5 }\end{array}$ & $\begin{array}{c}\text { Est_3 BE } \\
(\mathrm{MeV}) \\
\text { Relation-6 }\end{array}$ \\
\hline 50 & 50 & 100 & 116 & 812.3 & 810.1 & 813.0 & 834.8 & 833.7 & 843.5 \\
\hline 50 & 51 & 101 & 116 & 825.2 & 823.1 & 825.7 & 845.5 & 844.5 & 854.0 \\
\hline 50 & 52 & 102 & 116 & 839.9 & 838.0 & 840.2 & 856.0 & 855.0 & 864.2 \\
\hline 50 & 53 & 103 & 116 & 852.0 & 850.1 & 852.1 & 866.4 & 865.4 & 874.2 \\
\hline 50 & 54 & 104 & 116 & 865.8 & 864.1 & 865.7 & 876.6 & 875.6 & 884.0 \\
\hline 50 & 55 & 105 & 116 & 877.0 & 875.4 & 876.9 & 886.6 & 885.6 & 893.6 \\
\hline 50 & 56 & 106 & 116 & 890.0 & 888.6 & 889.8 & 896.4 & 895.4 & 903.0 \\
\hline 50 & 57 & 107 & 116 & 900.5 & 899.1 & 900.2 & 906.0 & 905.0 & 912.1 \\
\hline
\end{tabular}




\begin{tabular}{|c|c|c|c|c|c|c|c|c|c|}
\hline 50 & 58 & 108 & 116 & 912.7 & 911.5 & 912.4 & 915.4 & 914.5 & 921.1 \\
\hline 50 & 59 & 109 & 116 & 922.4 & 921.2 & 922.1 & 924.7 & $\begin{array}{l}923.8 \\
\end{array}$ & 929.8 \\
\hline 50 & 60 & 110 & 116 & 933.9 & 932.8 & 933.6 & 933.8 & 932.9 & 938.3 \\
\hline 50 & 61 & 111 & 116 & 942.9 & 941.9 & 942.7 & 942.6 & 941.8 & 946.7 \\
\hline 50 & 62 & 112 & 116 & $\begin{array}{l}953.8 \\
\end{array}$ & 952.8 & 953.6 & 951.4 & 950.5 & $\begin{array}{l}954.8 \\
\end{array}$ \\
\hline 50 & 63 & 113 & 116 & 962.1 & 961.1 & 962.0 & 959.9 & 959.0 & 962.8 \\
\hline 50 & 64 & 114 & 116 & 972.3 & 971.4 & 972.3 & 968.2 & 967.4 & 970.5 \\
\hline 50 & 65 & 115 & 116 & 980.0 & 979.1 & 980.1 & 976.4 & 975.6 & 978.1 \\
\hline 50 & 66 & 116 & 116 & 989.5 & $\begin{array}{l}988.8 \\
\end{array}$ & 989.8 & 984.4 & 983.6 & 985.5 \\
\hline 50 & 67 & 117 & 116 & 996.7 & 995.9 & 997.1 & 992.2 & 991.4 & 992.6 \\
\hline 50 & 68 & 118 & 116 & 1005.6 & 1004.9 & 1006.1 & 999.8 & 999.0 & 999.7 \\
\hline 50 & 69 & 119 & 116 & 1012.2 & 1011.4 & 1012.9 & 1007.2 & 1006.5 & 1006.5 \\
\hline 50 & 70 & 120 & 116 & 1020.6 & 1019.9 & 1021.4 & 1014.5 & 1013.7 & 1013.1 \\
\hline 50 & 71 & 121 & 116 & 1026.7 & 1025.9 & 1027.7 & 1021.5 & 1020.8 & 1019.6 \\
\hline 50 & 72 & 122 & 116 & 1034.5 & 1033.8 & 1035.7 & 1028.4 & 1027.7 & 1025.9 \\
\hline 50 & 73 & 123 & 116 & 1040.1 & 1039.3 & 1041.5 & 1035.1 & 1034.4 & 1032.0 \\
\hline 50 & 74 & 124 & 116 & 1047.4 & 1046.7 & 1049.0 & 1041.6 & 1041.0 & 1038.0 \\
\hline 50 & 75 & 125 & 116 & 1052.5 & 1051.6 & 1054.3 & 1048.0 & 1047.3 & 1043.8 \\
\hline 50 & 76 & 126 & 116 & 1059.3 & 1058.5 & 1061.3 & 1054.1 & 1053.5 & 1049.4 \\
\hline 50 & 77 & 127 & 116 & 1063.9 & 1063.1 & 1066.1 & 1060.1 & 1059.5 & 1054.8 \\
\hline 50 & 78 & 128 & 116 & 1070.3 & 1069.5 & 1072.7 & 1065.9 & 1065.3 & 1060.1 \\
\hline 50 & 79 & 129 & 116 & 1074.5 & 1073.5 & 1077.1 & 1071.5 & 1070.9 & 1065.3 \\
\hline 50 & 80 & 130 & 116 & 1080.5 & 1079.5 & 1083.3 & 1076.9 & 1076.3 & 1070.2 \\
\hline 50 & 81 & 131 & 116 & 1084.2 & 1083.2 & 1087.3 & 1082.1 & 1081.6 & 1075.1 \\
\hline 50 & 82 & 132 & 116 & 1089.8 & 1088.7 & 1093.0 & 1087.2 & 1086.7 & 1079.7 \\
\hline 50 & 83 & 133 & 116 & 1093.1 & 1091.9 & 1096.6 & 1092.1 & 1091.6 & 1084.2 \\
\hline 50 & 84 & 134 & 116 & 1098.3 & 1097.0 & 1101.9 & 1096.8 & 1096.3 & 1088.6 \\
\hline 50 & 85 & 135 & 116 & 1101.2 & 1099.9 & 1105.1 & 1101.3 & 1100.8 & 1092.8 \\
\hline 50 & 86 & 136 & 116 & 1106.0 & 1104.6 & 1110.0 & 1105.6 & 1105.1 & 1096.8 \\
\hline 50 & 87 & 137 & 116 & 1108.6 & 1107.1 & 1112.9 & 1109.7 & 1109.3 & 1100.7 \\
\hline 50 & 88 & 138 & 116 & 1113.0 & 1111.5 & 1117.4 & 1113.7 & 1113.3 & 1104.5 \\
\hline 50 & 89 & 139 & 116 & 1115.3 & 1113.6 & 1119.9 & 1117.5 & 1117.1 & 1108.1 \\
\hline 50 & 90 & 140 & 116 & 1119.3 & 1117.6 & 1124.1 & 1121.1 & 1120.7 & 1111.5 \\
\hline 50 & 91 & 141 & 116 & 1121.2 & 1119.4 & 1126.3 & 1124.5 & 1124.1 & 1114.9 \\
\hline 50 & 92 & 142 & 116 & 1124.9 & 1123.0 & 1130.1 & 1127.7 & 1127.4 & 1118.0 \\
\hline 50 & 93 & 143 & 116 & 1126.5 & 1124.5 & 1132.0 & 1130.8 & 1130.5 & 1121.1 \\
\hline 50 & 94 & 144 & 116 & 1129.9 & 1127.8 & 1135.5 & 1133.7 & 1133.3 & 1124.0 \\
\hline 50 & 95 & 145 & 116 & 1131.2 & 1129.0 & 1137.0 & 1136.3 & 1136.0 & 1126.7 \\
\hline 50 & 96 & 146 & 116 & 1134.3 & 1131.9 & 1140.2 & 1138.8 & 1138.6 & 1129.4 \\
\hline 50 & 97 & 147 & 116 & 1135.3 & 1132.8 & 1141.4 & 1141.2 & 1140.9 & 1131.9 \\
\hline 50 & 98 & 148 & 116 & 1138.0 & 1135.5 & 1144.3 & 1143.3 & 1143.1 & 1134.2 \\
\hline 50 & 99 & 149 & 116 & 1138.8 & 1136.1 & 1145.2 & 1145.3 & 1145.0 & 1136.4 \\
\hline 50 & 100 & 150 & 116 & 1141.3 & 1138.5 & 1147.8 & 1147.0 & 1146.8 & 1138.5 \\
\hline 50 & 101 & 151 & 116 & 1141.7 & 1138.8 & 1148.5 & 1148.6 & 1148.5 & 1140.5 \\
\hline 50 & 102 & 152 & 116 & 1143.9 & 1140.9 & 1150.8 & 1150.1 & 1149.9 & 1142.3 \\
\hline 50 & 103 & 153 & 116 & 1144.2 & 1140.9 & 1151.1 & 1151.3 & 1151.1 & 1144.0 \\
\hline 50 & 104 & 154 & 116 & 1146.1 & 1142.8 & 1153.2 & 1152.3 & 1152.2 & 1145.6 \\
\hline 50 & 105 & 155 & 116 & 1146.1 & 1142.5 & 1153.3 & 1153.2 & 1153.1 & 1147.0 \\
\hline 50 & 106 & 156 & 116 & 1147.7 & 1144.1 & 1155.0 & 1153.9 & 1153.8 & 1148.3 \\
\hline 50 & 107 & 157 & 116 & 1147.5 & 1143.7 & 1154.9 & 1154.4 & 1154.3 & 1149.5 \\
\hline 50 & 108 & 158 & 116 & 1148.9 & 1145.0 & 1156.4 & 1154.7 & 1154.6 & 1150.6 \\
\hline 50 & 109 & 159 & 116 & 1148.4 & 1144.3 & 1156.0 & 1154.8 & 1154.8 & 1151.6 \\
\hline
\end{tabular}




\begin{tabular}{|l|l|l|l|l|l|l|l|l|l|}
50 & 110 & 160 & 116 & 1149.6 & 1145.4 & 1157.2 & 1154.8 & 1154.8 & 1152.4 \\
\hline 50 & 111 & 161 & 116 & 1148.9 & 1144.5 & 1156.6 & 1154.6 & 1154.6 & 1153.1 \\
\hline 50 & 112 & 162 & 116 & 1149.8 & 1145.3 & 1157.6 & 1154.2 & 1154.2 & 1153.7 \\
\hline 50 & 113 & 163 & 116 & 1148.9 & 1144.2 & 1156.8 & 1153.6 & 1153.6 & 1154.1 \\
\hline 50 & 114 & 164 & 116 & 1149.7 & 1144.8 & 1157.5 & 1152.8 & 1152.9 & 1154.5 \\
\hline
\end{tabular}

115

\section{Second term of relation (6)}

We noticed that, reduction of binding energy is greatly influenced by electroweak interaction. Clearly speaking, second term plays a major role in reducing nuclear binding energy compared to other terms. This can be confirmed with relations (4) and (5). Number of non-participating nucleons seems to increase with increasing mass number. For ${ }_{92} \mathrm{U}^{238}$, number of non-participating nucleons are 48 and corresponding reduction in binding energy is $490 \mathrm{MeV}$. This is approximately $27 \%$ of total binding energy.

\section{First part of the third term of relation (6)}

Considering the third term, it constitutes two sub terms. First sub term is $\left(0.118 \frac{\left(N^{2}-Z^{2}\right)}{\sqrt{A A_{s}}}\right)$ and the second sub term is $\left(\frac{\left(A-A_{s}\right)^{2}}{\sqrt{A A_{s}}}\right)$. First sub term is almost similar to the Ghahramany's proposed second term, $\frac{\left(N^{2}-Z^{2}\right)}{3 Z}$. Multiplying and dividing relation (1) by 3 and ignoring $\delta(N-Z)$, it is possible to re-express it as,

$$
B E \cong\left\{A-\frac{\left(N^{2}-Z^{2}\right)}{9 Z}-3\right\}(9.9 \mathrm{MeV})
$$

It may be noted that, $\frac{\left(N^{2}-Z^{2}\right)}{9 Z} \cong \frac{1}{9}\left[\frac{\left(N^{2}-Z^{2}\right)}{Z}\right] \cong 0.11 \overline{1}\left[\frac{\left(N^{2}-Z^{2}\right)}{Z}\right]$. The number $\left(\frac{1}{9}\right) \cong 0.11 \overline{1}$ almost resembles the currently believed strong coupling constant [11,12], $\alpha_{s} \cong 0.118$. Keeping this coincidence in view we tried to replace $\left(\frac{1}{9}\right)$ with $\alpha_{s}$. By doing so, it is possible to bring strong interaction characteristics into nuclear binding energy scheme. It needs a serious review.

\section{Second part of the third term of relation (6)}

It is quite interesting to note that, second sub term of the third term plays a nice role in reducing the binding energy of isotopes of $Z$ about its stable mass number. Clearly speaking, on both side of the selected stable mass number of $Z$, it helps in curve bending with reference to mainstream semi empirical mass formulae. 


\section{Fourth term of relation (6)}

With trial-error, we have introduced the fourth term in place of the third term of the Ghahramany's integrated nuclear model. Even though, its contribution in reducing binding energy of medium and heavy atomic nuclides is minute, for light atomic nuclides, its role is considerable. It needs a review and refinement with respect to actual binding energy.

\section{Discussion on the binding energy coefficient}

Unique binding energy coefficient can be understood in two possible ways. One way is based on the basic nuclear potential and reciprocal of the strong coupling constant[12]. Second way is to consider the average rest energy of up and down quarks [12].

Method-1: Considering nuclear potential and strong coupling constant, unique binding energy coefficient can be expressed as,

$$
\begin{gathered}
B_{0} \cong\left(\frac{1}{\alpha_{s}}\right) \frac{e^{2}}{4 \pi \varepsilon_{0} R_{0}} \cong(10.0 \text { to } 10.4) \mathrm{MeV} \\
\text { where, }\left\{\begin{array}{l}
\alpha_{s} \cong 0.115 \text { to } 0.12 \\
R_{0} \cong 1.20 \mathrm{fm}
\end{array}\right.
\end{gathered}
$$

Method-2: Considering up and down quark masses, unique binding energy coefficient can be expressed as,

$$
\left.\begin{array}{c}
B_{0} \cong \frac{\left(2 m_{u} c^{2}+m_{d} c^{2}\right)+\left(m_{u} c^{2}+2 m_{d} c^{2}\right)}{2} \\
\cong \frac{3}{2}\left(m_{u} c^{2}+m_{d} c^{2}\right) \cong 10.26 \mathrm{MeV} \\
\text { where }\left\{\begin{array}{l}
m_{u} c^{2} \cong 2.16_{-0.26}^{+0.49} \mathrm{MeV} \\
m_{d} c^{2} \cong 4.68_{-0.17}^{+0.48} \mathrm{MeV}
\end{array}\right\}
\end{array}\right\}
$$

Combining relations (11) and (12),

$$
\alpha_{s} \cong \frac{2 e^{2}}{12 \pi \varepsilon_{0} R_{0}\left(m_{u}+m_{d}\right) c^{2}}
$$

\section{Conclusion}

Ghahramany's integrated nuclear binding energy formula can be modified to include strong and electroweak interaction features with a single energy coefficient. Based on relations (4), (5) and (6) and data presented in Table 1, we are very confident to say that, electroweak interaction plays a vital role in fixing nuclear binding energy and it is absolutely possible to understand nuclear binding energy with one unique energy coefficient. In this context, to explore the ground reality, 
we appeal the science community to look into these kinds of unified and simplified nuclear binding energy models.

\section{Conflict of Interest}

The authors declare no conflict of interest in this paper.

\section{Data Availability statement}

Energy coefficients of relations (7), (8) and (9) can be obtained from the following URL's.

1) Relation-7: https://fdocuments.in/document/nuclear-and-particle-physics.html

2) Relation-8: https://en.wikipedia.org/wiki/Semi-empirical_mass_formula

3) Relation-9: http://oregonstate.edu/instruct/ch374/ch418518/lecture3-1.pdf

\section{Acknowledgements}

Authors are very much thankful to Dr. N. Ghahramany and team for their intuitive and heuristic contributions in this most advanced field of nuclear research. Author Seshavatharam is indebted to professors shri M. Nagaphani Sarma, Chairman, shri K.V. Krishna Murthy, founder Chairman, Institute of Scientific Research in Vedas (I-SERVE), Hyderabad, India and Shri K.V.R.S. Murthy, former scientist IICT (CSIR), Govt. of India, Director, Research and Development, I-SERVE, for their valuable guidance and great support in developing this subject.

\section{References}

[1] Royer G, Subercaze A (2013) Coefficients of different macro-microscopic mass formulae from the AME2012 atomic mass evaluation. Nuclear Physics A 917: 1-14

[2] Cht Mavrodiev S, Deliyergiyev MA (2018) Modification of the nuclear landscape in the inverse problem framework using the generalized Bethe-Weizsäcker mass formula. Int. J. Mod. Phys. E 27: 1850015

[3] Xia, X. W., Lim, Y., Zhao, P. W., Liang, H. Z., Qu, X. Y., Chen, Y., \&Meng, J. (2018) The limits of the nuclear landscape explored by the relativistic continuum Hartree-Bogoliubov theory. Atomic Data and Nuclear Data Tables, 121, 1-215.

[4] Möller, P., Sierk, A. J., Ichikawa, T., \& Sagawa, H. (2016) Nuclear ground-state masses and deformations: FRDM (2012). Atomic Data and Nuclear Data Tables, 109, 1-204.

[5] N.Ghahramany, H.Sarafraza, E. Yazdankish. (2013) Stability and Mass Parabola in Integrated Nuclear Model.Universal Journal of Physics and Application 1(1): 18-25,

[6] Ghahramany, N., Gharaati, S., \& Ghanaatian, M. (2012) New approach to nuclear binding energy in integrated nuclear model. Journal of Theoretical and Applied Physics, 6(1), 3.

[7] Ghahramany N, Sh Gharaati, Ghanaatian M, Hora H (2011) New scheme of nuclide and nuclear binding energy from quark-like model. Iranian Journal of Science \& Technology. A3: 201-208.

[8] N. Ghahramani et al. (2008) Nuclear magic numbers based on a quark like model is compared with the Boltzmann distribution model from nuclear abundance in the universe and low energy nuclear reactions. Physics Essays, 21, 3, 200-206. 
220 [9] N. Ghahramany, M. Ghanaatian and M. Hooshmand. (2007) Quark-Gluon Plasma Model and 221 Origin of Magic Numbers.Iranian Physical Journal, 1-2, 35-38.

222 [10] Seshavatharam UVS and Lakshminarayana S. (2021) On the Combined Role of Strong and 223 Electroweak Interactions in Understanding Nuclear Binding Energy Scheme. Mapana Journal 224 of Sciences, 20(1),1-18.

225 [11] Seshavatharam UVS, Lakshminarayana S (2019) On The Role of Nuclear Quantum Gravity 226 In Understanding Nuclear Stability Range of $Z=2$ to 118. J. Nucl. Phys. Mat. Sci. Rad. A 7(1): 43-51.

[12] Tanabashi et al (2018) Review of Particle Physics: Particle Data Group. Phy. Rev. D. 98 


\section{Supplementary Files}

This is a list of supplementary files associated with this preprint. Click to download.

- enbe21Aug2021.pdf 